\title{
Intercambio de saberes en torno a las prácticas de crianza entre la sala cuna y la familia ${ }^{1}$
}

\author{
Knowledge About Child Rearing Practices: Exchange Between Nursery and Family²
}

Intercâmbio de saberes em torno das práticas de cuidado entre o berçário e a família

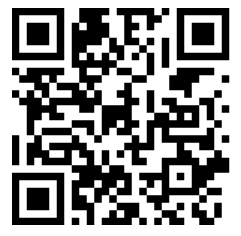

\author{
Astrid Gabriela Bravo-Soto 4 \\ Universidad Católica de la Santísima Concepción \\ Departamento de Curriculum y Evaluación, Facultad de Educación \\ Concepción, Chile \\ abravo@ucsc.cl
}

\begin{abstract}
Recibido • Received • Recebido: 13 / 10 / 2016
Corregido • Revised • Revisado: 13 / 04 / 2018

Aceptado•Accepted • Aprovado: 05/ 06 / 2018
\end{abstract}

\begin{abstract}
Resumen: Se presenta un estudio de caso realizado en Chile, orientado a conocer cómo se produce el intercambio de saberes en torno a las prácticas de crianza entre la sala cuna y las familias y cómo se utiliza este saber para enriquecer la propuesta curricular del nivel. Los datos se recogieron mediante análisis de documentos técnicos, observación del trabajo diario en sala y entrevistas en profundidad al personal del centro y a las madres. Se realizó un análisis de contenido cualitativo y se triangularon las distintas fuentes. Los hallazgos muestran diferencias en las prácticas relacionadas con las prioridades que se establecen en cada uno de los contextos, las dinámicas que se dan en el transcurso diario, y los parámetros para juzgarlas. Se observan algunos intercambios con respecto a las prácticas de alimentación y de la siesta. Se requiere avanzar en la construcción de propuestas que rescaten los saberes de las familias, así como ampliar la mirada con respecto a lo que se selecciona para ser transmitido a sus bebés.
\end{abstract}

Palabras claves: Crianza del niño y de la niña; educación de la primera infancia; rol de la familia.

\footnotetext{
${ }^{1}$ Estudio financiado por el proyecto DIN 09/2014. UCSC.

${ }^{2}$ This study was financed by the Project DIN 09/2014, UCSC.

${ }^{3}$ Estudo financiado pelo projeto DIN 09/2014, UCSC.

${ }^{4}$ Educadora de Párvulos, Magíster en Educación de la Universidad de Concepción. Actualmente, se desempeña en el Departamento de Currículum y Evaluación de la Facultad de Educación de la Universidad Católica de la Santísima Concepción. Imparte docencia en las asignaturas de Teorías Curriculares y Curriculum de la Educación Parvularia Primer y Segundo Ciclo. Su línea de investigación y sus publicaciones se vinculan con el ámbito de la educación de niños y niñas de cero a tres años.
} 
doi: http://dx.doi.org/10.15359/ree.22-3.5

URL: http://www.una.ac.cr/educare

CORREO: educare@una.cr

\begin{abstract}
This article presents a case study conducted in Chile. The purpose of this study was to know how the exchange of knowledge on rearing practices occurs between the nursery and families, and how this knowledge is used to enrich the curriculum proposal for the level. Data were collected through document analysis, observation of babies during the workday, and in-depth interviews with the mothers and the staff of the center. Content analysis and triangulation of sources were carried out. The findings show differences in the practices related to the priorities established for each of the contexts, the dynamics that occur in the daily routine, and parameters for judging those differences. Some exchanges are observed concerning feeding practices and the siesta time. It is required to advance in the construction of curricular proposals to rescue families' knowledge, as well as to broaden the perspective regarding what is selected to be transmitted to the babies.
\end{abstract}

Keywords: Child rearing practices; early childhood education: family role.

Resumo: Apresenta-se um estudo de caso realizado no Chile, orientado a conhecer como é produzido o intercâmbio de saberes em torno das práticas de cuidado entre o berçário e as famílias e como esse saber é utilizado para enriquecer a proposta curricular do nível. Os dados foram coletados por meio de análise de documentos técnicos, observação do trabalho diário na sala e entrevistas em profundidade com a equipe do berçário e as mães. Realizou-se uma análise de conteúdo qualitativo e se triangularam a diferentes fontes. Os resultados mostram diferenças nas práticas relacionadas às prioridades que são estabelecidas em cada um dos contextos, as dinâmicas que ocorrem no curso diário e os parâmetros para julgá-los. Observam-se alguns intercâmbios com respeito às práticas de alimentação e do descanso da tarde. Requer-se avançar na construção de propostas que resgatem os saberes das famílias, bem como ampliar a visão relacionada ao que se seleciona para ser transmitido a seus bebês.

Palavras-chave: Cuidado das crianças; educação da primeira infância; papel da família.

\title{
Introducción
}

Actualmente, una de las prioridades del nivel de educación parvularia se relaciona con la ampliación de la cobertura de atención para los niños y niñas de cero a tres años. Específicamente, se proyecta la creación de 90.000 cupos en centros parvularios en los niveles sala cuna, en 4.500 salas; y 34.000 cupos en los niveles medio, en aproximadamente 1.400 salas (Faverio, 2014). Según la Encuesta Casen 2013 (Ministerio de Desarrollo Social, Gobierno de Chile, 2013), la cobertura neta en el tramo cero a tres años es solo de un $28,4 \%$. En lo que respecta a sala cuna, un 18, 4 \% de niños y niñas menores de dos años asiste al nivel (Comunidad Mujer, 2016).

Si bien la incorporación de bebés a la sala cuna no es masiva, muchas familias envían a sus hijos e hijas a este nivel, especialmente, aquellas que están incorporadas al ámbito laboral. Para sus bebés, esto implica la inmersión en un escenario desconocido donde hay nuevas personas, objetos y situaciones; mientras que, para sus familias, una gran preocupación con respecto a los cuidados y educación que recibirán sus hijos e hijas. 
En ambos contextos, sala cuna y hogar, se transmiten saberes a la niñez. En el hogar, las familias educan y socializan a su bebé a través de sus prácticas de crianza, al cuidarle, alimentarle, mudarle, entre otras; influenciadas principalmente, por sus creencias y tradiciones. Mientras que, en la institución educativa, se repiten muchas de estas acciones y se intencionan aprendizajes a partir de distintos referentes contenidos en los diseños curriculares nacionales, en las propuestas del centro, y en aquellos que surgen de los contextos donde se desarrolla la acción educativa. En el ámbito de la educación formal, la selección de los saberes, así como las orientaciones para su transmisión se realizan, generalmente, por especialistas a partir de referentes teóricos, y se desconoce, muchas veces, el aporte que pueden realizar otros actores como las familias y las comunidades que son parte de los contextos de desarrollo infantil. En virtud de la especificidad que tiene la educación de bebés, resulta fundamental rescatar aquellos saberes que provienen del ámbito familiar y comunitario, especialmente, lo que concierne a las prácticas de crianza, y elaborar propuestas curriculares que validen este tipo de saber.

El presente artículo expone parte de los resultados de un estudio de caso desarrollado en una sala cuna. Se describen las principales características de algunas prácticas que predominan en el contexto de la sala cuna y en el hogar, la forma y tipo de intercambio de saberes sobre las prácticas de crianza que se produce entre ambos contextos y se destacan, además, algunas diferencias y similitudes en estas prácticas.

\section{Referentes conceptuales}

\section{Prácticas de crianza}

Al hablar de prácticas de crianza, generalmente, nos remitimos primero a lo que ocurre en el contexto hogareño, a lo que las madres y padres realizan cuando crían a sus hijos e hijas.

Aguirre (2000), en una revisión del concepto, señala:

La práctica de crianza es lo que efectivamente hacen los adultos encargados de cuidar a los niños. Son acciones que se orientan a garantizar la supervivencia del infante, a favorecer su crecimiento, desarrollo psicosocial, y aprendizaje de conocimientos; son acciones que, una vez inducidas, le permiten reconocer e interpretar su entorno. (p. 28)

Para Violante (2008a), la crianza constituye un proceso educativo, dirigido al niño o a la niña, que se hace a través de acciones cotidianas como alimentarle, mudarle, hacerle dormir y jugar, donde se va transmitiendo un conjunto de saberes sociales propios y valorados por la comunidad, con el objeto de contribuir a su desarrollo personal y social. 
doi: http://dx.doi.org/10.15359/ree.22-3.5

URL: http://www.una.ac.cr/educare

CORREO: educare@una.cr

De acuerdo con los estudios reseñados, la crianza se orienta a la supervivencia y desarrollo de la niñez, implica prácticas sociales diarias basadas en los conocimientos y creencias que tienen las personas adultas que les crían.

Violante (2008b) señala que la crianza se realiza en distintos escenarios tales como hogares, jardines infantiles y otros espacios donde habitan niños y niñas, y personas adultas compartiendo interacciones permanentemente. En estos escenarios, los sujetos adultos educan y socializan a la niñez más pequeña, transmitiéndoles su cultura. Si se establecen relaciones positivas e intercambios entre los distintos escenarios, se aumenta el potencial para favorecer el desarrollo infantil. Entendida de este modo, la crianza constituye una responsabilidad que no solo pertenece al ámbito privado, sino también al público, pues tiene que ver con la responsabilidad de distintas instituciones, más allá de la familiar. Consecuentemente, cuando las familias envían a sus hijos e hijas a una sala cuna están compartiendo la crianza de su bebé.

Al ser las prácticas de crianza acciones vinculadas a un contexto, los grupos adultos encargados de cuidar y educar al niño o a la niña promueven ciertos comportamientos específicos por sobre otros, pues están influenciados por parámetros culturales "la noción de crianza presenta fuertes componentes culturales y que, por lo tanto, los sentidos y significados de las tareas dependerán de la cosmovisión que se sustente" (Cottet, Pérez, Martínez, Sena, 2009, p. 53). En este sentido, no habría prácticas universales para la crianza, sino que estas estarían relacionadas con las características de los diferentes contextos en que se realizan, aspecto que resulta clave para quienes comparten la responsabilidad del cuidado y educación de parvularios. Por lo tanto, la familia y el centro educativo al constituir distintos escenarios de crianza, deberían intercambiar saberes en torno a las prácticas de crianza de bebés, con el objeto de influirse mutuamente.

Cuando un bebé o una bebé asiste a un centro educativo transita de un contexto familiar conocido, donde prevalecen ciertas prácticas de crianza, a un contexto educativo especializado, donde las acciones se organizan en función de propósitos pedagógicos. Ynoub (2008) menciona que las actividades de una jornada familiar no se planifican, tampoco se fundamentan teóricamente, ni se exponen a miradas externas, más bien se revisan en el transcurso diario a partir de creencias, tradiciones y valores; asimismo tienen una fuerte base afectiva, con un predominio del vínculo sobre la tarea. En virtud de ello, los centros educativos deberían favorecer formas de organización que recuperen los modos en que se desarrollan las acciones cotidianamente, evitando modalidades escolarizadas (Soto y Vasta, 2008).

De acuerdo con Willis y Ricciuti (2000), existen aspectos fundamentales en la atención de bebés, como mantener con él o ella una interacción estable, afectiva y lo más individualizada posible; ofrecerle variadas experiencias que le permitan ir descubriendo progresivamente su entorno, y un intercambio permanente con su familia. En la misma línea, Moreau (2001) señala que las experiencias 
de desarrollo para el niño o niña serán mejores si las personas adultas que le cuidan, interactúan con él o ella en forma cariñosa y estable, posibilitándole acceso al mundo simbólico por medio de juegos, cuentos, objetos u otros elementos que son parte de su cultura. Lo anterior implica que las experiencias educativas que se proponen a bebés en el nivel sala cuna deberían caracterizarse por interacciones cotidianas, estables y cálidas, donde se incluyan variados elementos culturales; y también, por un intercambio permanente con sus familias con el objeto de recoger los saberes que estas poseen con respecto a la crianza. Tal como señalan Cottet et al. (2009):

Una sana y buena crianza supone la construcción de acuerdos que trasciendan la esfera de lo privado. La crianza compartida desde una dimensión de lo público interpela lo privado para redefinir el cuidado y los derechos de los niños y niñas. (p. 57)

\section{Orientaciones curriculares para el nivel sala cuna}

La educación parvularia es el primer nivel de la educación chilena, les recibe desde los primeros meses de vida hasta su ingreso a la educación básica. De cero a dos años, asisten a la sala cuna y tienen atención de personal profesional y técnico. El personal educador de parvularios es profesional especializado en su atención y dentro de sus funciones están formar a niños y niñas, junto a la familia; diseñar, implementar y evaluar currículos; así como seleccionar procesos de enseñanza y dinamizar a la comunidad educativa en función de los aprendizajes de la población parvularia (Ministerio de Educación [Mineduc], Gobierno de Chile, 2005).

Las propuestas educativas que realiza el personal educador para bebés que asisten al nivel sala cuna consideran, como principal referente, el documento de las Bases Curriculares de la Educación Parvularia, elaborado por el Ministerio de Educación. Una de las principales ideas fuerza que sustenta esta propuesta es enfatizar el rol de la familia como el principal agente educativo y concebir al primer nivel educativo como una instancia que acompaña y apoya esta labor:

En la familia se establecen los primeros y más importantes vínculos afectivos y, a través de ella, la niña y el niño incorporan las pautas y hábitos de su grupo social y cultural, desarrollando los primeros aprendizajes y realizando sus primeras contribuciones como integrantes activos. (Mineduc, Gobierno de Chile, 2005, p. 13)

Este referente destaca, además, la importancia de los diferentes contextos para el desarrollo de la población parvularia, especialmente, aquellos que corresponden a la cultura de pertenencia, pues favorecen su identidad y autoestima. Asimismo, señala que respetar y valorizar la diversidad étnica, lingüística y cultural de las diferentes comunidades significa reconocerlas e incorporarlas en la construcción e implementación curricular (Mineduc, Gobierno de Chile, 2005). 
doi: http://dx.doi.org/10.15359/ree.22-3.5

URL: http://www.una.ac.cr/educare

CORREO: educare@una.cr

Gimeno (2010) destaca que el currículo se relaciona con una selección regulada de los contenidos a enseñar y aprender en el contexto escolar. Por lo tanto, implica una decisión acerca de qué conocimientos serán seleccionados con la intención de ser transmitidos al estudiantado en los diferentes niveles educativos. En lo que respecta a las instituciones que educan a bebés, Marotta (2009) recalca que los conocimientos que se transmiten en este contexto constituyen contenidos, pues son enseñados y aprendidos en una institución educativa y obedecen a una intencionalidad. Sin embargo, estos contenidos tienen una especificidad distinta, comprenden "aspectos que en edades más avanzadas no son considerados como saberes escolares a ser abordados" (Pitluk, 2011, p. 60). Según Soto y Violante (2005), estos se asocian más al "hacer"y sus principales referentes deberían ser los saberes culturales, las formas de crianza hogareña, los aspectos del desarrollo y los saberes disciplinares desde lo actitudinal.

Considerar las formas de crianza hogareña significa que parte de lo que se transmite a bebés, incluiría cómo las personas adultas interactúan con esta niñez, alimentan, mudan, hacen dormir, en general, todas las acciones que realizan para transmitir saberes a sus hijos e hijas.

El desarrollo curricular supone la puesta en práctica de una propuesta elaborada a nivel nacional que es interpretada por el centro educativo. También, involucra la incorporación de un conjunto de elementos que surgen de los propios contextos en que se desarrolla la acción educativa para enriquecer las propuestas. En este sentido, la elaboración de propuestas curriculares en el nivel sala cuna debería considerar aquellos conocimientos que poseen las familias sobre la crianza, tanto sus características como las creencias y los saberes que las sustentan, del mismo modo, el intercambio de perspectivas en torno a ello.

En cada contexto, las prácticas de crianza se hacen desde lo que se considera apropiado dentro de un ámbito específico. En lo que respecta a la familia, pesan las tradiciones y creencias arraigadas en la comunidad a la que se pertenece. Mientras, que en establecimientos educativos, centros de salud $u$ otras instituciones abocadas al trabajo con la infancia, influyen referentes teóricos provenientes de distintas disciplinas y algunos acuerdos internacionales, como la Convención de los Derechos del Niño, que actualmente tiene una gran influencia en las políticas y programas dirigidos a la infancia. Intercambiar saberes con respecto a las prácticas de crianza exige comprender desde dónde se juzgan las acciones que se realizan, de igual forma, apertura y diálogo con respecto a perspectivas distintas a las propias.

\section{Metodología}

El objetivo de la investigación era conocer cómo se está produciendo el intercambio de saberes en torno a las prácticas de crianza entre los centros educativos y las familias que envían a sus hijos e hijas a la sala cuna y cómo este saber se utiliza para enriquecer las propuestas curriculares. Específicamente, interesaba caracterizar las prácticas de crianza utilizadas en cada 
contexto, identificar algunos elementos comunes o distintivos, y analizar cómo se intercambian y utilizan estos saberes para enriquecer las propuestas curriculares.

Acorde con los objetivos, la investigación se abordó desde un paradigma cualitativo, ya que interesaba conocer sobre la realidad social y cultural desde el punto de vista de sus protagonistas (Vieytes, 2004a). Tuvo un carácter exploratorio y descriptivo (Hernández, Fernández y Baptista, 2003), pues el ámbito curricular en el nivel sala cuna corresponde a un área muy poco explorada. Estudios nacionales incluyen, preferentemente, temáticas relacionadas con la línea de apego y con calidad del ambiente de cuidado y desarrollo infantil. En cuanto a los estudios sobre las prácticas de crianza, estos han sido abordados, preferentemente, desde el campo de la sicología y la sociología.

Se realizó un estudio de caso, lo que equivale a una recopilación e interpretación detallada de información sobre un individuo, institución, o movimiento social particular. Específicamente, correspondió a un estudio de caso múltiple, pues la unidad de análisis correspondió a más de un caso en diferentes contextos (Vieytes, 2004b). Para conocer las prácticas de crianza que predominaban, tanto en el contexto del centro educativo, como en los hogares de los niños y niñas que asisten a la sala cuna, fue necesario permanecer un periodo prolongado en el terreno y entrevistar a diferentes actores vinculados al proceso educativo (personal y familias), observar las prácticas que prevalecen diariamente en el aula y revisar documentos y registros que son utilizados para orientar y desarrollar el trabajo pedagógico con bebés.

Los criterios de selección correspondieron a: jardines infantiles con atención en el nivel sala cuna con dos años mínimo de funcionamiento; ubicados en distintas comunas, rural y urbana; de fácil acceso, se consideró tanto cercanía geográfica, como la disponibilidad de los centros para realizar el estudio.

\section{Técnicas e instrumentos}

Se aplicaron entrevistas en profundidad, "encuentros reiterados, cara a cara, entre el investigador y los informantes, ... dirigidos hacia la comprensión ... que tienen los informantes respecto de sus vidas, experiencias o situaciones" (Taylor y Bogdan (1986), citado en Ruiz, 2003b, p. 167). Se realizó un análisis de documentos (Simons, 2011). Además, se efectuó una observación en terreno, es decir, una inmersión en el caso de estudio, pero no intrusiva, la que Ruiz (2003a) define como una observación panorámica no participante, pues implica que quien investiga no participa por completo de la vida social del grupo que observa, sino que participa como persona observadora; y tanto la persona investigadora como las personas observadas conocen los objetivos y planes de la persona investigadora. 
doi: http://dx.doi.org/10.15359/ree.22-3.5

URL: http://www.una.ac.cr/educare

CORREO: educare@una.cr

Los instrumentos se diseñaron previamente, se elaboraron guías para la observación de prácticas de crianza y pautas para las entrevistas. Se realizó una aplicación piloto de los instrumentos diseñados en un centro educativo que contaba con atención en el nivel sala cuna. Después de esta aplicación, los instrumentos fueron ajustados.

\section{Participantes}

Se entrevistó a la directora del establecimiento y al personal que estaba a cargo de los niños y niñas de la sala cuna, una educadora de parvulario y tres del personal técnico. Asimismo, a los sujetos apoderados de bebés, 12 madres de un total de 18 .

\section{Procedimiento}

El estudio se inició estableciendo contacto formal con una institución de educación parvularia de cobertura nacional, se informó el propósito del proyecto y se solicitó acceso a un centro ubicado en una zona rural. Una vez que la institución facilitó el acceso a un jardín infantil, se tomó contacto con la directora para agendar reunión y dar a conocer el proyecto de investigación. Esta instancia permitió recoger información sobre las características y funcionamiento general del centro, establecer algunos acuerdos con respecto a la forma en que se recogerían los datos y planificar las primeras visitas.

El trabajo de recolección de datos se prolongó por más de tres meses, entre septiembre y diciembre, y contempló la revisión de documentación a nivel de jardín infantil, así como de la sala del nivel sala cuna. Se revisó el referente curricular de la institución, el proyecto educativo del jardín infantil, las planificaciones de la sala cuna (anual y periódicas); además, una serie de registros escritos utilizados por el personal: pedagógico, bienestar, accidentes, higiene, vulneración, y los del trabajo con familia (actividades, reuniones y talleres).

En lo que respecta a las entrevistas, todas se realizaron en un clima grato, lo que permitió una hora de conversación con cada una de las integrantes del equipo de la sala. En cuanto a las personas apoderadas de los bebés que asistían al nivel sala cuna, se acordó con la educadora una estrategia para poder entrevistar a la mayoría. Estos encuentros fueron grabados, previo consentimiento informado, en sesiones que duraron entre 45 y 60 minutos. Todas las entrevistas fueron realizadas por la investigadora principal.

Con respecto a las observaciones, estas se realizaron en dos oportunidades. Se observó todo lo que acontecía durante la jornada diaria en la sala cuna, al comenzar el proceso de recolección de datos, y luego se repitió cuando se había entrevistado al personal de la sala cuna. 


\section{Análisis de datos}

Se realizó un análisis de contenido, forma de recoger información para leerla de manera objetiva, sistemática, replicable y válida; para luego, analizarla y elaborar alguna teoría o generalización sobre ella (Ruiz, 2003c). Se transcribieron las entrevistas grabadas, se digitaron los registros de las observaciones y las notas de la revisión de los documentos del centro. Se leyeron cuidadosamente todos estos datos, se codificaron y se organizaron en matrices según categorías y subcategorías. Estas se definieron a priori, y en la medida que progresaba el análisis, se ajustaron y se incluyeron otras, de tipo emergente. Esta categorización fue revisada constantemente durante el procedimiento de análisis.

En cuanto al rigor metodológico, se triangularon fuentes documentales con las entrevistas y las observaciones. Además, se realizaron validaciones con los propios informantes. Una vez que las entrevistas fueron transcritas, se volvió al centro para que el personal de la sala cuna tuviera la posibilidad de revisarlas y señalar observaciones. Después del análisis preliminar, se difundieron los hallazgos en el centro para su validación.

\section{Hallazgos}

El primer caso de estudio correspondió a un jardín infantil, con más de cinco años de funcionamiento, ubicado en una comuna rural, y financiado con fondos públicos. Dependiente de una institución de cobertura nacional, desde donde emanan orientaciones que se contextualizan en los proyectos educativos de cada establecimiento. El nivel que se indagó correspondió a una sala cuna heterogénea que atendía a 18 niños y niñas, de lunes a viernes, desde las 8.30 a las 16.30 hrs; a cargo de una profesional educadora de parvularios y de tres sujetos del personal técnico. El centro estaba emplazado en un sector habitacional, construido para las familias damnificadas por el terremoto del 27 de febrero de 2010, y muy cercano a una caleta de pescadores. Las familias cuyos bebés asistían al centro vivían en el sector y sus principales actividades laborales se relacionaban con la pesca artesanal y con el programa PRO Empleo 5 .

A continuación, se presentan los principales hallazgos del Caso 1, según dimensión, categoría y subcategoría.

\footnotetext{
${ }^{5}$ Programa destinado a la inserción laboral de los grupos más vulnerables del país y a otorgar una fuente laboral en lugares donde se haya experimentado alguna contracción de la demanda debido a una emergencia.
} 
doi: http://dx.doi.org/10.15359/ree.22-3.5

URL: http://www.una.ac.cr/educare

CORREO: educare@una.cr

\section{Dimensión prácticas de crianza}

\section{Concepto y valoración de las prácticas de crianza en la sala cuna}

Las prácticas de crianza son entendidas como las acciones que realizan las personas que conviven diariamente con el niño o la niña, orientadas a su cuidado y educación.

Involucra hábitos, involucra acciones diarias ... Adulto 1

...lo que uno hace acá en el jardín, lo que hace la familia en la casa, ... es como enseñar, ... es como orientar. Adulto 4

...responder a demandas de cuidado, protección y educación de los hijos/as de acuerdo a necesidades y también a la experiencia y crianza de las familias... Adulto 5

Las prácticas consideradas apropiadas están vinculadas con lo que establece la Convención de los Derechos del Niño, referente que orienta los protocolos que guían las acciones de las personas adultas en sala; y se asocian al bienestar general de bebés, como cuidado de su higiene. Mientras que las prácticas consideradas inapropiadas, se relacionan con falta de preocupación por la higiene y, en general, con cualquier situación que atente contra su bienestar y que vulnere alguno de sus derechos.

Una buena práctica por, ejemplo, para mí, es preocuparse en la parte de higiene del niño, también brindarle un entorno de bienestar dentro de lo posible en su casa. Adulto 2

\section{Características de las prácticas de crianza en la sala cuna}

\section{Interacciones entre el personal adulto y cada bebé}

Se caracterizan por una intencionalidad pedagógica, que incluye verbalizaciones acompañadas con gestos dirigidos a bebés con respecto a lo que se va realizando durante la jornada, se dan en un clima grato y con un tono afectivo. El personal se organiza con la modalidad de "un adulto principal", es decir, una persona adulta se hace cargo, preferentemente, de un grupo de niños y niñas de la sala para favorecer un contacto más cercano.

Se le explica que vamos a entrar a almorzar, que vamos a comer tal cosa, que hay que lavarse las manos y todo eso, ... Adulto 2 
Nosotros tratamos de crear un apego seguro en realidad, un apego seguro con todos los niños y que tengan sus tías de referencia y que se sientan apoyados por sus tías. Adulto 5

\section{Juegos y actividades}

Son, en su mayoría, planificados y propuestos por el sujeto adulto, se intencionan de acuerdo con los periodos estructurados durante la jornada y con los aprendizajes que se quieren favorecer acorde con el referente curricular nacional (formación personal y social, comunicación, relación con el medio natural y cultural), énfasis institucional (lenguaje verbal y pensamiento matemático), y local (seres vivos y su entorno).

Los niños no están por ahí volando por así decir, nosotros tenemos periodos y estos periodos tienen intencionalidad. Cada periodo, el encuentro, por ejemplo, que es donde los niños... nos saludamos, cómo llegamos, se hacen preguntas, la gracia es que el niño pequeño te responde. Luego está el jugar, ... Adulto 1

Las actividades son variadas como, por ejemplo, cantos con mímica, observación de libros y láminas, dibujo de trazos en papel, pintura con los dedos, juego con pelotas y juguetes de arrastre, manipulación y exploración de diversos objetos, juegos de desplazamiento y equilibrio. También, se incluyen actividades en el exterior con implementos. Las actividades están dirigidas, principalmente, a bebés que caminan (se trabaja aparte con la otra población más pequeña) y poseen un formato: se les ubica sentados o sentadas, se presenta material y se hace una invitación a realizar algunas acciones que finalizan, generalmente, cuando el personal adulto lo indica. El personal adulto verbaliza todas las acciones para favorecer el desarrollo del lenguaje verbal.

Se le muestran cubos grandes y cubos chicos y ahí se hace una actividad con eso, después la tía reparte cubos para cada uno y nosotros nos sentamos en grupitos y ahí lo vamos reforzando, grande -chico, le mostramos las características... Adulto 2

Los momentos para el desplazamiento, juego y exploración libre se dan, preferentemente, en los periodos de tránsito, cuando se espera el inicio de otro periodo de la jornada.

...en el momento en que llegan los niños, el niño que llega en el momento del encuentro, antes de que se reúnan todos, los niños sacan distintos materiales...

... son las instancias cuando son más libres entre comillas, donde pueden escoger un material, es como más individualmente, donde se fortalece el apego, es como la parte más íntima entre la tía y el niño... Adulto 3 
doi: http://dx.doi.org/10.15359/ree.22-3.5

URL: http://www.una.ac.cr/educare

CORREO: educare@una.cr

\section{Higiene y mudas}

Las mudas se realizan en periodos establecidos para ello, después de la alimentación, preferentemente. Sin embargo, los bebés y las bebés pueden mudarse en otros periodos, si así se requieren. Estas se realizan en el baño y en un mudador acondicionado para ello. Se sigue un protocolo centrado en su bienestar, que incluye tanto el resguardo de las condiciones de higiene, como interacciones orientadas a fortalecer el vínculo con el bebé o la bebé y a estimular su lenguaje verbal.

Al niño se le invita a la muda se dice ¿vamos a la muda?, se le invita, el niño viene animadamente a la muda, pero antes de eso, primero se tiene que privilegiar el ambiente donde se va a traer al niño, o sea, que yo tengo que colocar, tener desinfectado el mudador, lavarme las manos, debo tener mi indumentaria, colocar el paño, la toallita que ellos traen, tener a mano el paño que se le va a mudar, la ropa que se le pueda cambiar, todo, todo previamente antes... Adulto 3

Si se detecta algún problema vinculado a la higiene, existe un protocolo que incluye el procedimiento a seguir, registro, comunicación y seguimiento de la situación. Mientras una o dos personas adultas están mudando, las otras permanecen en la sala con el resto de bebés (periodo de transición). Las actividades de higiene también contemplan lavado de manos y cara, uso de servilleta y cepillado dental. Cada bebé es mudado por la persona adulta de referencia.

\section{Periodos de alimentación}

En estos periodos se sigue un protocolo que incluye ambientación, disponer mesas y sillas; resguardo de la higiene del lugar, uso de pechera, lavado de manos, creación de un clima grato y estrategias para que el grupo de bebés ingiera los alimentos. Si un bebé presenta dificultades, se utilizan recursos verbales y gestuales para apoyarlo; y si no recibe el alimento, no se le obliga a hacerlo. Durante el desayuno, el almuerzo y la once el bebé está a cargo de su persona adulta de referencia. Se incluyen señales y cantos para los periodos. La alimentación es acorde a la edad; incluye legumbres, carnes y pescado, vegetales, frutas y lácteos. Los grupos más pequeños comen papilla y los más grandes picado. Se evitan situaciones que involucren riesgo para la salud de cada bebé, por ejemplo, alimentos que le provocan alergia.

\section{Periodos de siesta}

Están programados dentro de la jornada e incluyen una ambientación, poner música suave, oscurecer la sala y preparar las cunas y catres donde cada bebé duerme. La siesta es todos los días a la misma hora y se sigue la misma rutina. Los sujetos adultos están familiarizados con los hábitos y costumbres que tienen los grupos de bebés para dormir, algunos se quedan dormidos 
sin apoyo, mientras que otros necesitan que se les acaricie o se les dé unos pequeños golpecitos en la espalda para conciliar el sueño. La siesta dura entre una hora, y una hora y media, después se retoma la rutina con la ingesta de leche.

\section{Normas y disciplina}

El personal trabaja en forma cotidiana la inculcación de normas y el resguardo de la disciplina dentro del aula. Se pone énfasis en mantener el control del grupo, por medio de indicaciones y en un tono amable. Se insiste en la importancia de compartir y evitar riesgos.

...por ejemplo un niño, por el material. Le entrego el material y viene otro niño y viene a quitarle el material, entonces es ahí donde uno tiene que intervenir como tía, entonces se le dice ¿eh?, colocándose a su altura, mirándolo a los ojos al niño, se le dice: no le quites el material, comparte... Adulto 3

Se marca el periodo que se está desarrollando y se cautela que todo el grupo de bebés siga la rutina establecida. Las formas que se utilizan para advertir o llamar la atención del niño o niña son verbales "esto no se hace"; además, se dan explicaciones simples de lo que se debe hacer. Los comportamientos más reprobados son: empujar, morder o quitar un objeto a otro bebé.

\section{Uso de los espacios y materiales}

Con respecto a los espacios, preferentemente, se utiliza la sala y el hall. La infraestructura y el mobiliario son nuevos, la sala es amplia e iluminada. Los exteriores son utilizados, pero con menos frecuencia. Los materiales se incorporan con un propósito pedagógico, se ubican en áreas, a la vista y altura de cada bebé, y están clasificados dentro de contenedores. Varían en tamaño, textura y color, e incluyen, entre otros, pelotas, juegos de arrastre, encajes, legos, muñecos, figuras de plástico y género, libros, materiales para expresión plástica e instrumentos musicales. El espacio se va adaptando a la rutina, se incorpora mobiliario u otros elementos de acuerdo con la actividad. Los grupos de bebés no deambulan mucho por la sala, salvo en periodos de transición. En general, el uso de los espacios es dirigido por el personal adulto, así como la posibilidad de acceder a los materiales.

\section{Concepto y valoración de las prácticas de crianza en el hogar}

Las entrevistadas entienden las prácticas de crianza como la enseñanza diaria que dirigen hacia sus hijos e hijas. 
doi: http://dx.doi.org/10.15359/ree.22-3.5

URL: http://www.una.ac.cr/educare

CORREO: educare@una.cr

Pienso que ir enseñándole todos los días cosas, ... Madre 3

La enseñanza, darle una buena educación y que tengan buenos modales. Madre 2

En cuanto a prácticas consideradas apropiadas, se mencionan la preocupación que se demuestra por el bebé y las expresiones de afecto.

... el cariño yo creo, el cariño a ello, o sea el cien por ciento, preocuparse del niño de lo que le pasa o que le duele algo... Madre 2

Yo creo que estar presente no más po, si, en darle cariño, en abrazarlo, decirle que lo quiero mucho, ... Madre 9

Dentro de las prácticas consideradas inapropiadas, se señalan la falta de preocupación por la higiene del grupo de bebés, descuidarles, no tomarles en cuenta y dejarles sin compañía.

\section{Características de las prácticas de crianza en el hogar}

\section{Interacciones entre la persona adulta y su bebé}

Las entrevistadas dan cuenta de variadas formas de interacción con sus bebés, como gestos, expresiones verbales y corporales. Asimismo, destacan ser "muy de piel", se privilegia en todo momento el contacto físico y las demostraciones de afecto.

Con ella, abrazos, besos, andar así, haciendo cariño, dándole besos, abrazos, porque es muy así de piel, andar, sí, o que la tome en brazos, que se me arranque, que yo le digo ven y la abrazo, así, me entiende. Madre 1

Que a él le toco su pelito, le hago cariño o lo abrazo, nosotros le decimos, denos un abrazo y él, abraza, nos da besitos, ... Madre 2

\section{Juegos y actividades}

Los grupos de bebés participan en un amplio repertorio de juegos y actividades, entre ellas, actividades motoras como caminar por los alrededores, andar en triciclo, jugar a la pelota y con la saltarina, escuchar música y moverse. Se incluyen también actividades como dibujar, pintar, jugar con muñecas, animales, autos de plástico u objetos cotidianos; y ver televisión, especialmente, programación infantil. También se les invita a colaborar en pequeños quehaceres 
domésticos, como ayudar a ordenar, sacar y guardar objetos, trasladar objetos a la mesa cuando se toma once, entre otras. Asimismo, las madres señalan que estas instancias son aprovechadas para estimular el lenguaje de su bebé.

...por ejemplo, cosas, yo estoy barriendo y ella, o me pongo a limpiar los muebles y dejo el escobillón ahí, y ella se pone a barrer conmigo y empieza a barrer la alfombra a hacer como que barre, porque no barre mucho, ahí se pone como a ayudarme a hacer tal cosa, igual que a ordenar la ropa, yo la dejo ordenar... Madre 10

Se aprecia que los grupos de bebés participan, frecuentemente, en actividades familiares y comunitarias, como almorzar o cenar, asistir a la iglesia, visitar la caleta, ir a la cancha de futbol o ir a la feria.

Tratamos de estar todos, si vamos a iglesia, vamos con ella; en la casa, si hay alguna cosa, yo si salgo, con ella a todo, si vamos por ejemplo, los sábados vamos a la feria, ahí vamos todos a la feria, tratamos de incluirla en todas,... Madre 1

Salimos al cerro, salimos a caminar a la playa, eso hacemos harto. Tenemos un tiempo en el día desocupado, un rato,..., así que sacamos a los perros al cerro, jugamos, llevamos pelota, así que nos divertimos harto en eso, tenemos harta vida familiar entre nosotros, y ella está siempre incluida... Madre 9

\section{Mudas e higiene}

La muda se realiza cuando el bebé o la bebé lo requiere, se revisa varias veces durante el día. Se realiza en un contexto de intercambio, el sujeto adulto juega y conversa con su bebé.

...él es bueno pa' orinar, entonces yo ahí le cambio al tiro el paño, antes de quedarse dormido le cambio el pañito, cosa que no se me vaya a cocer, lo mudo cada dos horas, tres horas, porque igual para dar del cuerpo también hace harto, si, es que yo lo noto al tiro porque lo reviso, lo estoy revisando constantemente ,... Madre 1

... solamente cuando se hace, cuando yo creo que está muy hecho pipi ahí le cambio, pero no le ando cambiando a cada rato tampoco, es que él es grande ya, y aparte que no se hace mucho... Madre 3

Juego con él, le canto, él se ríe, juego con las piernitas, a él le encanta que le hagan fiesta, si, si, le gusta a él... Madre 6 
doi: http://dx.doi.org/10.15359/ree.22-3.5

URL: http://www.una.ac.cr/educare

CORREO: educare@una.cr

Se realiza, preferentemente, encima de una cama donde se ubica un mudador. Se invita a participar al bebé para que traiga su paño u otro implemento, y se le incentiva a que avise. Como práctica de higiene se incluye, además, baño, lavado de manos y cara, uso de peineta y cepillado dental. Al bebé lo muda la persona adulta que le cuida en casa, en la mayoría de los casos la madre, pero también participa el padre u otro sujeto adulto de la familia.

\section{Periodos de alimentación}

Estos periodos incluyen ciertas rutinas que se repiten todos los días de lunes a viernes, el bebé o la bebé toma leche (mamadera o pecho) al levantarse, y después, al regreso de la sala cuna. Durante la tarde, se intenta seguir un mismo horario (once o cena en algunos casos). Mientras que, los fines de semana, el almuerzo se intenta servir en el mismo horario de la sala cuna, alrededor de las 11.30. El bebé se sienta en una silla de comer. El tipo de alimento es variado: carnes, pescado, legumbres, vegetales, frutas, lácteos, incluso en algunos hogares se incluyen mariscos. Se permite la ingesta de golosinas, principalmente, como premio. La comida se les da molida o picada. La ingesta se da en un clima grato y se promueve su autonomía. Si no come solo o sola, se le entrega una cuchara para que colabore; si lo hace, una persona adulta le supervisa. Si no quiere comer, no se le obliga, y se utilizan distintas estrategias como juegos, darle la comida u ofrecerle un lácteo en su reemplazo.

\section{Periodos de siesta}

Se tiende a seguir un mismo horario, después del almuerzo. En promedio cada bebé duerme una hora, pero si se extiende a dos o más, les dejan dormir. Asimismo, si quieren dormir en otro periodo se les permite. Como estrategias para facilitar el sueño, se le pasa el chupete, un peluche, un objeto de su agrado como el "tuto" y algunas madres, todavía utilizan el pecho para hacerle dormir.

\section{Normas y disciplina}

Las entrevistadas mencionan la necesidad de poner límites a sus bebés y lo indispensable que es hacerlo. Se les llama la atención en forma verbal cuando hacen algo indebido, como pelear o morder a otro bebé y se les conversa por qué no debe hacerlo o se utilizan gestos como "expresión de enojo". Algunas entrevistadas, señalan sentir culpa cuando llaman la atención a su bebé, reconocen que a veces utilizan un tono fuerte y exagerado al hacerlo, y que realizan algunas prácticas como lavarle la cara cuando está mañoso. Generalmente, se utilizan premios cuando se porta bien. 


\section{Espacios y materiales}

Con respecto a la utilización de los espacios, se aprecia que sus bebés permanecen en espacios interiores y exteriores. En lo que respecta a interiores se utilizan, preferentemente, dormitorios y el living. En cuanto a los exteriores, la mayoría de las familias adapta los patios, techando y habilitando para el juego. Se aprovechan otros espacios cercanos, canchas, áreas verdes y paseos a la playa. En ocasiones, sus bebés que se desplazan juegan en la calle, pero con la supervisión adulta. En cuanto a los materiales, se cuenta con implementos para el juego como: pelotas, muñecas, autos, diversas figuras, plásticas o de género; triciclos y juegos de arrastre. También, en algunos hogares, cuentan con hojas de papel, lápices, encajes y rompecabezas.

\section{Elementos comunes o distintivos en las prácticas de crianza evidenciadas en la sala cuna y en el hogar}

Considerando el propósito del estudio, orientado a conocer cómo se produce el intercambio de saberes en torno a las prácticas de crianza entre la sala cuna y las familias y cómo se utiliza este saber para enriquecer la propuesta curricular del nivel, se identifican algunos elementos comunes o distintivos en las prácticas de crianza.

\section{Elementos comunes}

Se aprecia bastante similitud respecto de lo que entienden por prácticas de crianza. En ambos contextos refieren a estas como acciones cotidianas, que realizan las personas adultas, orientadas a cuidar y enseñar algo al niño o a la niña, asimismo, se observa que la preocupación por su bienestar general constituye una "buena práctica".

En lo que respecta a las características de las prácticas, las principales coincidencias se dan en los periodos de siesta, especialmente, en los horarios en que esta se desarrolla. En cuanto a los periodos de alimentación, se observan similitudes en el tipo de alimentación, en las prácticas adultas para alimentarlos, así como en los horarios. En ambos contextos existe preocupación por el establecimiento de normas de comportamiento y en la forma de hacerlo: se llama la atención en forma verbal, se le conversa y explica, orientando su comportamiento. Asimismo, se potencia el desarrollo del lenguaje verbal.

\section{Elementos distintivos}

Se aprecian algunas diferencias, relacionadas con los referentes, las prioridades que se establecen en cada uno de los contextos y con las dinámicas que se dan en el transcurso diario. 
doi: http://dx.doi.org/10.15359/ree.22-3.5

URL: http://www.una.ac.cr/educare

CORREO: educare@una.cr

En el contexto "sala cuna" se tiene como referente para juzgar las prácticas la perspectiva de los derechos de infancia, toda situación considerada vulnerabilidad para el niño o la niña se juzga desde la perspectiva de sus derechos, mientras que, en el hogar, no existe un referente específico. En lo que respecta a las interacciones entre personas adultos y bebés, para las madres resulta clave la creación del vínculo, el contacto físico y la demostración de afecto, mientras que en el centro prima la intencionalidad pedagógica en la interacción.

En lo que respecta a los juegos y actividades, se observa que, en el contexto hogareño, sus bebés participan en muchas actividades cotidianas como ayudar a la persona adulta en tareas del hogar o involucrarse en actividades familiares como ir a misa, a la cancha, entre otras. Mientras que en el centro, participan en actividades planificadas por el personal adulto, dentro de un formato establecido (presentación de materiales e indicaciones) y con la intención de favorecer un aprendizaje específico.

En cuanto a las mudas, en el contexto hogareño estas no se programan, se hacen según requerimiento de cada bebé, revisándole periódicamente durante la jornada diaria; mientras que en la sala cuna se establecen horarios para ello. En lo que respecta a espacios, en el centro se consideran preferentemente interiores, los que se implementan y organizan con una intencionalidad pedagógica. En cambio, en el hogar, se utilizan distintos espacios, sin que necesariamente se encuentren implementados para sus requerimientos.

\section{Dimensión intercambio de saberes}

\section{Percepción que tiene el personal de la sala cuna sobre las familias y el entorno}

\section{Cultura local}

Se reconocen algunos rasgos de identidad en las familias, visibles en sus hábitos y costumbres y vinculados con la cultura de vida en una caleta de pescadores. Las entrevistadas señalan que las familias utilizan un vocabulario menos formal que el utilizado en el jardín infantil, más ligado a sus actividades cotidianas. Por otra parte, perciben una cultura machista, especialmente en lo que respecta al rol de la mujer como cuidadora de los hijos e hijas. También, reconocen que los horarios de bebés están más influenciados por el acontecer diario en la caleta, son más flexibles $y$, a veces, pueden llegar a ser perjudiciales, como permanecer sin dormirse y comer después de las 10 de la noche. Valoran fuertemente la preocupación que las familias demuestran por sus hijos e hijas y el compromiso con su educación, las caracterizan como personas "muy de piel", pues privilegian el contacto físico y las expresiones de cariño. Reconocen que muchas de sus prácticas están arraigadas en la tradición. 


\section{Rol del jardín}

Se destaca la función pedagógica de la institución y se reconoce la labor orientadora que esta tiene con respecto a la educación de la población parvularia, así como la necesidad de trabajar en forma articulada con las familias.

\section{Rol de las familias}

Se reconoce a la familia como agente educativo y se espera que esta apoye lo que el jardín realiza como labor educativa. Su rol principal sería la crianza de los hijos e hijas en un clima de afecto.

\section{Percepción de las familias con respecto a su rol y al del jardín infantil}

\section{Rol del jardín infantil}

Las madres entrevistadas valoran el rol pedagógico, señalando que la institución enseña a los niños y niñas las reglas, a estar con otras personas y a un quehacer más autónomo. Reconocen que sus hijos e hijas aprenden al asistir al centro educativo.

Aprenden, le enseñan cosas, hábitos, ... Madre 2

\section{Rol de las familias}

Destacan que su principal rol es la crianza de sus hijos e hijas, es decir, guiarles en su desarrollo en un ambiente de afecto. Algunas madres señalan que comparten muchas veces el rol del jardín infantil, pues ellas también enseñan a sus bebés.

\section{Tipo y forma de intercambio entre el jardín infantil y las familias}

\section{Las formas de intercambio entre las familias y el centro educativo}

Se orientan a la creación de vínculos y lazos de confianza. Se utilizan variadas formas de intercambio, tanto formales como informales. Dentro de las formales, está el uso de distintos tipos de registro, algunos son diarios y otros, acorde a la situación que necesita ser registrada. Se visualizan dos elementos importantes en el uso de estos registros: uno, dejar evidencia escrita sobre situaciones que acontecen en el centro y que respaldan el procedimiento utilizado, por ejemplo, algo relacionado con la higiene del niño/a; otro, realizar seguimiento con respecto a lo que acontece en el ámbito pedagógico, pues diariamente se escribe y comenta sobre las 
doi: http://dx.doi.org/10.15359/ree.22-3.5

URL: http://www.una.ac.cr/educare

CORREO: educare@una.cr

actividades que se realizan en el aula. Muchos de los registros utilizados contemplan también un espacio para que las familias expresen sus opiniones hacia el centro. Además de los registros escritos, están las entrevistas que se realizan al inicio del periodo escolar con el fin de recabar información y durante el proceso, para resolver alguna situación puntual. Durante el año, también se programan distintos talleres y reuniones mensuales. Asimismo, se dan instancias menos formales como el momento en que los adultos retiran a los bebés del centro, el cual se aprovecha para intercambiar en forma más directa con la madre o el padre del bebé.

\section{Tipo de intercambio}

Las prácticas de crianza son parte de los intercambios habituales entre el centro y la casa. Especialmente, lo que refiere a los periodos de alimentación, siesta y muda.

De primera era como muy difícil hacer dormir a mi niña, entonces yo tenía que decirle: tía lo que pasa que cuando era más chiquitita yo tomaba a mi niña en brazo y me la ponía aquí en mi pecho y bailaba con ella, ahí la niña se quedaba dormida, entonces aquí como no sabían, yo le tuve que contar eso a las tías para la hora de la siesta... Madre 1

Se desarrollan talleres con las familias, donde se aborda el tema de la crianza: estilos, pautas, prácticas, normas y límites, entre otros; apoyados por material que elabora la institución. En lo que respecta a las prácticas de alimentación, se intercambia sobre el tipo de alimentación y la forma de hacerlo. Lo mismo ocurre con la siesta, con respecto a horarios y estrategias para hacer dormir a los bebés y a las bebés. Las normas y la disciplina también son parte del intercambio, se conversa sobre el comportamiento de cada bebé, tanto en la casa como en la sala cuna, y lo que se hace para evitar conductas no apropiadas. Se intercambia sobre los aprendizajes, principalmente a través de los informes que se envían a la casa con respecto a los avances que han tenido. Otros intercambios están relacionados con el funcionamiento general del centro.

\section{Tipo de saberes incorporados en la propuesta curricular}

\section{Orientaciones incorporadas en documentos}

Se evidencian orientaciones para el desarrollo curricular plasmadas en documentos institucionales que hacen explícita la importancia y necesidad de incorporar los saberes que aportan las familias y la comunidad con respecto a la crianza de los niños y niñas. En lo que respecta al proyecto educativo (piloto) que elaboró el centro, se recogen algunos intereses de las familias en cuanto a temáticas a tratar y se visibiliza alguna referencia a la cultura local, una caleta de población pescadora, en la prioridad que se establece con respecto del núcleo

Astrid Gabriela Bravo-Soto

Los artículos de la Revista Electrónica Educare del Centro de Investigación y Docencia en Educación de la Universidad Nacional, Costa Rica, se comparten bajo términos de la Licencia Creative Commons: Reconocimiento, No Comercial, Sin Obra Derivada 3.0 Costa Rica. Las autorizaciones adicionales a las aquí delimitadas se pueden obtener en el correo: educare@una.cr 
de aprendizaje "Seres vivos y su entorno". En las planificaciones de aula no se evidencia algún aporte de las familias con respecto a las prácticas que realizan con sus hijos e hijas.

\section{Implementación curricular}

Se aprecia que se ha producido algún intercambio con respecto a los modos en que se hacen algunas acciones dirigidas a los grupos de bebés. Esto se observa especialmente en las prácticas de alimentación y en los periodos de siesta con respecto a horarios y formas de realizarlas.

Así también, la práctica del sujeto adulto principal, un grupo de bebés a cargo de una misma persona para favorecer relaciones más cercanas, que si bien no está basada directamente en el contexto del jardín infantil, sí contempla una característica de los ambientes hogareños, una interacción más individualizada con el bebé.

Por otra parte, se develan aspectos clave en lo declarado por el personal, que podrían contribuir a enriquecer las propuestas a nivel de aula. Uno, relacionado con que se debería aprovechar más la identidad que tienen las familias con su entorno, una caleta de pescadores, para incorporarla al trabajo que se realiza en el aula; otro, que los saberes que poseen las familias en torno a la crianza, tendrían que ser el punto de partida para potenciar y orientar las acciones dirigidas hacia sus bebés en la sala cuna.

\section{Conclusiones y discusión}

En cuanto a los objetivos del estudio, se pudo caracterizar las prácticas de crianza utilizadas en cada contexto. En lo que respecta a la sala cuna, la Convención de los Derechos del Niño constituye el principal referente para establecer los protocolos que orientan las acciones de los sujetos adultos en los diferentes periodos de la jornada diaria, donde el bienestar de la niñez es una prioridad en las prácticas de alimentación, siesta, mudas e higiene, así como en los momentos en que se instalan aprendizajes más específicos. En el contexto de los hogares, los referentes para orientar las acciones adultas provienen de distintos ámbitos. Se aprecian prácticas arraigadas en la tradición que conviven con nuevas influencias recibidas, especialmente del centro educativo.

Respecto de elementos comunes o distintivos en las prácticas, se comparte lo que se entiende por práctica de crianza y algunas características de estas, especialmente aquellas que se vinculan con la alimentación y la siesta de sus bebés. Se observan algunas diferencias, relacionadas con las prioridades que se establecen en cada uno de los contextos y con las dinámicas que se dan en el transcurso diario. En la sala cuna las acciones se anticipan en función de los aprendizajes que se quieren favorecer, se contemplan dinámicas más estructuradas con periodos establecidos, espacios organizados acordes con los requerimientos de bebés 
doi: http://dx.doi.org/10.15359/ree.22-3.5

URL: http://www.una.ac.cr/educare

CORREO: educare@una.cr

y actividades programadas; mientras que, en los hogares, las acciones no se anticipan, las dinámicas diarias son más flexibles en lo que respecta al tiempo y el uso de los espacios, asimismo se prioriza el vínculo afectivo con sus bebés.

En relación con el intercambio de saberes entre la sala cuna y las familias en torno a las prácticas de crianza y su utilización para el enriquecimiento de las propuestas curriculares, se observan algunos intercambios en cuanto a prácticas de alimentación y siesta de bebés, los que se han realizado tanto por medio de instancias formales, comunicaciones diarias, talleres, entrevistas o reuniones; como informales, conversaciones que se dan entre el "adulto principal" y familiar que retira a su bebé del centro. Asimismo, se evidencia, en lo declarado por el personal y en las orientaciones plasmadas en los documentos institucionales, un reconocimiento de los saberes que poseen las familias sobre la crianza, así como la necesidad de incorporarlos en la implementación del currículo. Sin embargo, estos saberes todavía son escasamente aprovechados para enriquecer las propuestas curriculares.

Algunos aspectos a discutir se relacionan con la perspectiva desde dónde se juzgan las prácticas de crianza, pues esta podría dificultar el intercambio de saberes en torno a la crianza. En efecto, los Derechos del Niño constituyen el principal parámetro para juzgar una práctica en el contexto de la sala cuna, todo lo que se entiende por buena crianza se asocia con las acciones que realizan los seres adultos en función de procurar bienestar a la niñez desde la perspectiva de sus derechos. En cambio, en los hogares, muchas de las prácticas están fuertemente arraigadas en las creencias que han sido transmitidas de una generación a otra. Este aspecto es visible, por ejemplo, en prácticas de higiene, como el baño, lo que puede ser visto desde distintas perspectivas. Por una parte, bañarle poco podría estar basado en la creencia que se puede resfriar; por otra, la poca frecuencia podría ser considerada una situación que atenta contra su bienestar general y, por lo tanto, un cuestionamiento a lo que se hace en el contexto familiar.

Ellos piensan que si bañan mucho a la guagua se va a resfriar, entonces tienen cosas como de, como de abuelos, claro, entonces estamos como luchando con eso, entonces dicen pero tía sí,... yo le digo el niño transpiró todo el día, eso también le hace mal, si tampoco es tenerlo media hora en la tina, es solamente una agüita, porque los niños transpiran mucho, ... Adulto 5

Sin desconocer la relevancia de este referente incorporado en muchas políticas y programas dirigidos a la infancia, es indispensable dialogar desde los distintos contextos en que se crían bebés. Esto implica atender los compontes culturales de las prácticas, abrir la mirada a otras formas de entender la realidad y comprender que existen diferencias culturales en estas prácticas. Como señalan Cottet et al. (2009), los sentidos y significados que las personas adultas asignan a sus acciones dependen de la cosmovisión que sustenten. En este sentido, hay que considerar que las familias no consideran necesariamente los mismos referentes y 
muchas de sus prácticas están arraigadas en la tradición y en modos de vida diversos que sería indispensable conocer, con el objeto de poder intercambiar perspectivas y enriquecer lo que se realiza en cada uno de los contextos.

Otro aspecto a revisar es la tendencia a valorar más las orientaciones que provienen principalmente de la institución educativa, lo que es reforzado por las propias familias, quienes en general reconocen y valoran los saberes que les entrega el centro educativo más que sus propios saberes. Intercambiar perspectivas y experiencias con respecto a la crianza debería implicar un análisis del tipo de contribución que se espera de las familias y revisar el tipo de saberes que se validan en el contexto de la institución educativa. Si se considera que el currículo se relaciona con la selección de un conjunto de saberes a transmitir a los niños y niñas en el contexto escolar y también con la forma en que se transmiten estos, sería necesario remirar las prácticas que se dan en el aula. Si bien en los documentos institucionales se establecen orientaciones que validan otras formas de saber, como el que poseen las familias y las comunidades donde habitan, en la implementación curricular predominan los referentes teóricos. Este aspecto debiera ser revisado en los centros por medio de procesos reflexivos que permitan a los distintos actores involucrados en la crianza de bebés ampliar miradas y elaborar propuestas de buenas prácticas desde los diferentes contextos donde habitan los niños y las niñas, considerando que las familias y las comunidades son diversas y que pueden contribuir a enriquecer lo que se hace en el ámbito educativo especializado.

Una de las formas de alejarse de las modalidades escolarizadas (Soto y Vasta, 2008) es revisar las prácticas que se sostienen en el contexto de la educación formal a la luz de lo que ocurre en el contexto familiar y construir propuestas con la participación de distintos actores, para validar los saberes que provienen de referentes no teóricos. Esto permitiría rescatar las formas que tienen las familias de interactuar con sus bebés e incluirlas en las dinámicas diarias en el centro. Lo anterior, requiere una revisión del sentido de las propuestas nacionales y el fortalecimiento de la construcción curricular a nivel de centro.

Por último, se sugiere ampliar este tipo de estudios, considerando la especificidad que tiene la educación de bebés, caracterizada por personas adultas que interactúan diariamente con ellos y ellas para jugar, alimentarles, mudarles o hacerles dormir; mientras traspasan variados elementos de la cultura por medio de la forma en que realizan estas prácticas. Sería necesario indagar con respecto a la selección cultural que se plasma en el currículo oficial para la educación de bebés, revisar cómo se están construyendo las propuestas curriculares en los diferentes centros educativos, así como examinar lo que los grupos educadores de parvularios seleccionan como contenidos y cómo se transmiten estos. 
doi: http://dx.doi.org/10.15359/ree.22-3.5

URL: http://www.una.ac.cr/educare

CORREO: educare@una.cr

\section{Referencias}

Aguirre, E. (2000). Socialización y prácticas de crianza. En E. Aguirre y E. Durán (Auts.), Socialización: Prácticas de crianza y cuidado de la salud. Un estudio con familias y niños que inician su escolarización en Santa Fe de Bogotá (pp. 17-92). Colombia: Centro de Estudios Sociales. Bogotá: Universidad Nacional de Colombia. Recuperado de http://www.bdigital. unal.edu.co/1548/5/02CAPI01.pdf

Comunidad Mujer. (2016). Mujer y trabajo: Sala cuna, un derecho para madres y padres trabajadores. Serie Comunidad Mujer, 35, 1-11. Recuperado de http://www. comunidadmujer.cl/biblioteca-publicaciones/2016/03/mujer-y-trabajo-sala-cuna-underecho-para-madres-y-padres-trabajadores/

Cottet, S., Pérez, C., Martínez, G. y Sena, C. (2009). ¿Es posible la crianza compartida entre las familias y el jardín maternal? En E. Marotta, M. S. Rebagliati y C. Sena (Coords.), ¿Jardín maternal o educación maternal? Ecos de una experiencia de formación docente (pp. 47-58). Buenos Aires: Novedades Educativas.

Faverio, F. (2014). La propuesta presidencial de educación parvularia en cifras. Recuperado de http://achie.cl/ciie2014/3996271.pdf?file=docs/sesiones/399.pdf

Gimeno, J. (2010). ¿Qué significa el currículum? En J. Gimeno (Comp.), Saberes e incertidumbres sobre el currículum (pp. 21-44). Madrid: Morata.

Hernández, R., Fernández, C. y Baptista, P. (2003). Metodología de la Investigación (3a ed.). México: McGraw Hill Interamericana.

Marotta, E. (2009). La didáctica del jardín maternal entendida como construcción colectiva. En E. Marotta, M. S. Rebagliati y C. Sena (Coords.), ¿ Jardín maternal o educación maternal? Ecos de una experiencia de formación docente (pp. 77-86). Buenos Aires: Novedades Educativas.

Ministerio de Desarrollo Social, Gobierno de Chile. (2013). Encuesta de caracterización socioeconómica nacional. Chile: Autor. Recuperado de http://observatorio. ministeriodesarrollosocial.gob.cl/casen-multidimensional/casen/casen 2013.php

Ministerio de Educación, Gobierno de Chile. (2005). Bases curriculares de la educación parvularia. (Capítulo 1: Fundamentos). Chile: Autor. Recuperado de http://portales.mineduc. cl/usuarios/convivencia escolar/doc/201103041242340.Bases Curriculares de Educacion Parvularia 2001.pdf

Moreau, L. (2001). Lo individual y lo social en el desarrollo humano. En A. de León, A. Malajovic y L. Moreau (Auts.), Pensando la educación infantil (pp. 141-151). Barcelona: Octaedro. 
Pitluk, L. (2011). Educar en el jardín maternal: Enseñar y aprender de 0 a 3 años (2ª reimp.). Buenos Aires: Novedades Educativas.

Ruiz, J. I. (2003a). Capítulo 4: La observación. En J. I. Ruiz (Aut.), Metodología de la investigación cualitativa (3a ed., pp. 125-164). Bilbado: Deusto.

Ruiz, J. I. (2003b). Capítulo 5: La entrevista. En J. I. Ruiz (Aut.), Metodología de la investigación cualitativa ( $3^{\text {a }}$ ed., pp. 165-190). Bilbado: Deusto.

Ruiz, J. I. (2003c). Capítulo 6: Análisis de contenido. En J. I. Ruiz (Aut.), Metodología de la investigación cualitativa. (3a ed., pp. 191-214). Bilbado: Deusto.

Simons, H. (2011). Capítulo III: Escuchar, mirar, documentar: Métodos de la investigación con estudio de caso. En H. Simons (Aut.), El estudio de caso: Teoría y práctica (pp. 70-101). Madrid: Morata.

Soto, C. A. y Vasta, L. E. (2008). Del alfabeto cultural: Prácticas cotidianas y juegos tradicionales En C. Soto y R. Violante (Eds.), Pedagogía de la crianza: Un campo teórico en construcción (pp. 173-195). Buenos Aires: Paidós.

Soto, C. A. y Violante, R (2005). Enseñar contenidos en el jardín maternal: Una forma de compartir la crianza. En C. Soto y R. Violante (Comps.), En el jardín maternal. Investigaciones, reflexiones y propuestas. (pp. 31-65). Buenos Aires: Paidós.

Vieytes, R. (2004a). Metodología de la investigación en organizaciones, mercado y sociedad. Epistemología y técnicas (Cap. 20). Buenos Aires: Editorial de las Ciencias.

Vieytes, R. (2004b). Metodología de la Investigación en organizaciones, mercado y sociedad (Cap. 21). Buenos Aires: Editorial de las ciencias.

Violante, R. (2008a). Por qué pedagogía de la crianza. En C. Soto y R. Violante (Eds.), Pedagogía de la crianza: Un campo teórico en construcción (pp. 23-53). Buenos Aires: Paidós.

Violante, R. (2008b). Los escenarios de crianza en la educación de los niños pequeños. En C. Soto y R. Violante (Eds.), Pedagogía de la crianza: Un campo teórico en construcción (pp. 55-75). Buenos Aires: Paidós.

Ynoub, R. (2008). Maternar, paternar, ¿escolarizar? Algunas conceptualizaciones en torno a funciones y contextos socializadores. En C. Soto y R. Violante (Eds.), Pedagogía de la crianza: Un campo teórico en construcción (pp. 99-119). Buenos Aires: Paidós.

Willis, A. y Ricciuti, H. (2000). Orientaciones para la escuela infantil de cero a dos años (3aed.). Madrid: Morata. 